\title{
Resummation prescriptions and ambiguities in SCET vs. direct QCD: Higgs production as a case study
}

\author{
Marco Bonvini, ${ }^{a, 1}$ Stefano Forte, ${ }^{b, c}$ Giovanni Ridolfi $^{d}$ and Luca Rottoli ${ }^{b, 1}$ \\ ${ }^{a}$ Deutsches Elektronen-Synchroton, DESY, \\ Notkestraße 85, D-22603 Hamburg, Germany \\ ${ }^{b}$ Dipartimento di Fisica, Università di Milano, \\ Via Celoria 16, I-20133 Milano, Italy \\ ${ }^{c}$ INFN, Sezione di Milano, \\ Via Celoria 16, I-20133 Milano, Italy \\ ${ }^{d}$ Dipartimento di Fisica, Università di Genova and INFN, Sezione di Genova, \\ Via Dodecaneso 33, I-16146 Genova, Italy \\ E-mail: marco.bonvini@desy.de, stefano.forte@mi.infn.it, \\ giovanni.ridolfi@ge.infn.it, luca.rottoli@physics.ox.ac.uk
}

ABSTRACT: We perform a comparison of soft-gluon resummation in SCET vs. direct QCD (dQCD), using Higgs boson production in gluon fusion as a case study, with the goal of tracing the quantitative impact of each source of difference between the two approaches. We show that saddle-point methods enable a direct quantitative comparison despite the fact that the scale which is resummed in the two approaches is not the same. As a byproduct, we put in one-to-one analytic correspondence various features of either approach: specifically, we show how the SCET method for treating the Landau pole can be implemented in $\mathrm{dQCD}$, and how the resummation of the optimal partonic scale of dQCD can be implemented in SCET. We conclude that the main quantitative difference comes from power-suppressed subleading contributions, which could in fact be freely tuned in either approach, and not really characteristic of either. This conclusion holds for Higgs production in gluon fusion, but it is in fact generic for processes with similar kinematics. For Higgs production, everything else being equal, SCET resummation at NNLL in the BecherNeubert implementation leads to essentially no enhancement of the NNLO cross-section, unlike dQCD in the standard implementation of Catani et al.

KEYWORDS: QCD Phenomenology

ARXIV EPRINT: 1409.0864

\footnotetext{
${ }^{1}$ Current address: Rudolf Peierls Centre for Theoretical Physics, University of Oxford, 1 Keble Road, OX1 3NP, Oxford, U.K.
} 


\section{Contents}

1 Sudakov resummation: advantages and ambiguities $\quad 1$

2 Resummation: from dQCD to SCET 3

3 Factorization and saddle-point approximation $\quad 11$

4 Logarithmically subleading vs. power-suppressed differences: an assess$\begin{array}{ll}\text { ment } & 16\end{array}$

5 Summary and outlook $\quad 20$

\section{Sudakov resummation: advantages and ambiguities}

The current standard for the computation of hard processes at the LHC, such as Higgs production in gluon fusion $[1,2]$, is to improve fixed order computations through the inclusion of soft-gluon resummation. This is often advantageous even for processes which are far from threshold, where the contributions which are resummed would become of order one, because it may provide a good approximation to the first few missing higher order corrections: this is indeed what happens for Higgs production in gluon fusion [3, 4]. However, especially when dealing with processes which are far from threshold, alternative implementations of threshold resummation which differ by subleading terms lead to predictions which might differ by an amount which is comparable to the effect of the resummation itself. Comparison of different resummation prescriptions and methodologies may then be of considerable phenomenological interest.

A particularly relevant instance of this situation is the comparison of results obtained when resummation is performed through the direct use of perturbative QCD (direct QCD, or dQCD, henceforth), or using an effective field theory (soft-collinear effective theory, or SCET, henceforth). For example, in the case of Higgs in gluon fusion [5-7] the effect of resummation can be by a factor two larger according to whether resummation is performed using SCET or dQCD [6]. Whereas there are indications that this is likely related to powersuppressed corrections [6], it would be highly desirable to have a detailed quantitative understanding of the relation between the two approaches.

In recent years there has been an increasing interest in a deeper understanding of similarities and differences between the SCET and dQCD approach to resummation. Whereas in the SCET papers refs. $[6,8,9]$ a first exploration of the relationship between the two approaches was performed, in refs. [10, 11] expressions relating SCET and dQCD resummation for various choices of soft scale were presented in analytic form, and finally, in refs. $[12,13]$, the relationship between the two formalisms was traced to the way they handle the underlying soft-gluon factorization. 
The full machinery which is necessary for a detailed quantitative comparison is thus available: the comparison will be the subject of the present paper. The goal of this work is complementary to that of refs. [10-13]: there, the aim was to show the equivalence of SCET and dQCD resummation by classifying terms which are resummed in either approach in terms of logarithmic accuracy. Thus, in particular, in refs. $[10,11]$ it was shown that dQCD and SCET resummed expressions differ by subleading logarithms of the hadronic scale, under suitable assumptions on the parton luminosity. Here, we will trace each ingredient which enters either resummed expression, retaining all differences, both logarithmicallyand power-suppressed, and assess their impact. While refs. [10,11] focused on the invariant mass distribution of Drell-Yan pairs, and ref. [13] on event shapes, here we will consider specifically Higgs production in gluon fusion. We will however always display results for a wide range of values of the Higgs mass: much wider, in fact, than the acceptable physical Higgs mass range. The goal is to study the qualitative behaviour in a wide region which may be of interest for other processes (such as Drell-Yan), while providing quantitatively precise prediction for physical Higgs production.

Soft-gluon resummation for hadronic processes in dQCD, as derived in refs. [14-17], is almost universally performed using the formalism of ref. [18], which we will take as a reference for the dQCD approach. On the other hand, the SCET approach comes in many flavors, which differ not only in technical details but, more importantly, in the choice of scale which is being resummed. Here, we refer specifically to the SCET approach of refs. [5, 8, 9, 19], which builds upon the derivation from SCET [20-24] of soft-gluon resummation [25-28]. This choice is not only motivated by the widespread use of this approach, but also by the fact that, because it is based on momentum space (as opposed to Mellin space), and a hadronic (instead of partonic) resummed scale choice, this version of SCET resummation is in some sense maximally different from dQCD, and thus it will allow us to explore all facets of the difference between SCET and dQCD. Henceforth, we will refer to these two approaches as dQCD and SCET, for short.

The starting point of our paper is a derivation, presented in section 2, of the relation between resummed expressions in $\mathrm{dQCD}$ and SCET which shows how starting with the dQCD expression one can arrive at the SCET result through a step-by-step modification of the starting expression. This derivation has the interesting byproduct of showing how some features of the SCET approach (such as the removal of the Landau pole or the use of a hadronic scale) could be implemented in a dQCD approach without having to use SCET, and by simply manipulating the dQCD expression. It is also more powerful than the master formula relating SCET to dQCD of refs. [10, 11]: whereas that relation only allowed for a classification of the logarithmic order of the difference, this new derivation keeps track of all individual contributions which differ in the two approaches.

Because in the SCET approach considered here it is a hadronic scale which is being resummed, and not a partonic scale as in the dQCD approach, it may seem that a comparison can only be made at the hadronic level, and this indeed was the point of view taken in refs. [10-13], where the dependence of results on the parton luminosity was also discussed. However, in section 3 we show that a comparison at the level of partonic cross-sections can be done by means of a saddle-point method. This also allows us to assess the quantitative 
impact of a first obvious source of difference between the dQCD and SCET approaches, namely, that because of the choice of resumming a hadronic scale the resummed SCET expression for the hadronic cross-section does not respect the standard factorized form of QCD expressions, in that it does not have the form of a convolution between a parton luminosity and a partonic cross section, which reduces to an ordinary product upon taking a Mellin transform.

With this problem out of the way, in section 4 we can then compare the partonic cross-section as obtained in either approach. We will in particular discuss separately two classes of contributions, which correspond to individual steps in the procedure previously discussed in section 2 which takes from the dQCD to the SCET expression: subleading logarithmic terms, which arise as a consequence of the shift from a partonic to a hadronic resummed scale, and the specific choice of power-suppressed terms which characterizes the standard SCET expression in comparison to the standard dQCD expression.

Specifically we shall show that, at least with the scale choice of refs. [5, 8, 9, 19], power-suppressed terms are by far the dominant source of difference. For Higgs production in gluon fusion, this difference turns out to be comparable to the effect of the resummation itself: indeed, the NNLO+NNLL result for gluon fusion, which in ref. [2] using a dQCD approach is found to be of order of $10 \%$, is reduced to a negligible enhancement at the percent level if everything else is kept equal, but the SCET approach of refs. [6, 7] is used instead, with the difference entirely due to power suppressed terms.

This is an interesting conclusion, because such terms are, in fact, unrelated to the choice of resumming via SCET or dQCD; rather, they may be freely tuned in either approach. So the difference is large indeed, but not intrinsically related to the use of dQCD vs. SCET, and specifically not to the treatment of the Landau pole, or the choice of resumming a hadronic instead of partonic scale. Rather, this is yet another instance of the fact that large ambiguities may come from power suppressed terms when resummation is used to improve perturbative predictions away from the threshold region, as already pointed out by us some time ago [29] in the context of Drell-Yan production. In the specific context of gluon fusion, it has been known for a long time [30] that some choices of power suppressed terms lead to an improved agreement of the truncation of resummed results with known fixed-order results. More recently, the large size of power-suppressed ambiguities to the soft approximation to Higgs production in gluon fusion computed at $\mathrm{N}^{3} \mathrm{LO}$ was pointed out in ref. [31], and a general systematic way of optimizing such corrections was suggested in ref. [3]. We shall briefly comment on the implications of this in our concluding section 5 .

\section{Resummation: from dQCD to SCET}

In this section we present a step-by-step argument which takes from the dQCD to the SCET form of the resummed cross-section. We will give our argument with specific reference to Higgs boson production in gluon fusion, up to NNLL accuracy.

We define the dimensionless cross-section $\sigma$ in terms of the physical cross section $\sigma_{\text {Higgs }}$ as

$$
\sigma\left(\tau, m_{H}^{2}\right)=\frac{1}{\tau \sigma_{0}} \sigma_{\mathrm{Higgs}}\left(\tau, m_{H}^{2}\right)=\int_{\tau}^{1} \frac{d z}{z} C\left(z, m_{H}^{2}\right) \mathcal{L}\left(\frac{\tau}{z}\right)
$$


where $\sigma_{0}$ is the leading-order partonic cross section for the process $g g \rightarrow H, m_{H}$ is the Higgs mass, $\tau=\frac{m_{H}^{2}}{s}$ and $s$ is the hadronic center-of-mass energy squared, $\mathcal{L}$ is the gluon luminosity, and $C$ a dimensionless coefficient function related to the partonic cross section. For simplicity, we do not show the dependence on factorization and renormalization scales, which we choose to be equal to $m_{H}$ throughout the paper.

The quantity in eq. (2.1) factorizes upon Mellin transformation with respect to $\tau$ :

$$
\boldsymbol{\sigma}\left(N, m_{H}^{2}\right)=\int_{0}^{1} d \tau \tau^{N-1} \sigma\left(\tau, m_{H}^{2}\right)=\boldsymbol{C}\left(N, m_{H}^{2}\right) \mathcal{L}(N)
$$

where

$$
\boldsymbol{C}\left(N, m_{H}^{2}\right)=\int_{0}^{1} d z z^{N-1} C\left(z, m_{H}^{2}\right) ; \quad \mathcal{L}(N)=\int_{0}^{1} d z z^{N-1} \mathcal{L}(z) .
$$

Because of the importance of keeping track of the difference between Mellin space and momentum space, we refrain from the common abuse of notation (of which we were specifically guilty in refs. $[10,11]$ ) whereby the same notation is used for a function and its Mellin transform; rather, we will follow the convention that a function and its Mellin transform are denoted with the same symbol, but with the Mellin transform in boldface.

The resummed $N$-space coefficient function in dQCD can be written as

$$
C^{\mathrm{dQCD}}\left(N, m_{H}^{2}\right)=\hat{g}_{0}\left(\alpha_{\mathrm{S}}\left(m_{H}^{2}\right)\right) \exp \hat{S}^{\mathrm{dQCD}}\left(m_{H}^{2}, \frac{m_{H}^{2}}{\bar{N}^{2}}\right)
$$

where $\bar{N}=N e^{\gamma_{E}}$ and

$$
\hat{S}^{\mathrm{dQCD}}\left(\mu_{1}^{2}, \mu_{2}^{2}\right)=\int_{\mu_{1}^{2}}^{\mu_{2}^{2}} \frac{d \mu^{2}}{\mu^{2}}\left[A\left(\alpha_{\mathrm{S}}\left(\mu^{2}\right)\right) \ln \frac{m_{H}^{2}}{\mu^{2} \bar{N}^{2}}+\hat{D}\left(\alpha_{\mathrm{S}}\left(\mu^{2}\right)\right)\right] .
$$

For later convenience, the two arguments of $\hat{S}^{\mathrm{dQCD}}$ have been chosen to be the two integration bounds.

The functions $\hat{g}_{0}\left(\alpha_{\mathrm{S}}\right), A\left(\alpha_{\mathrm{S}}\right)$ and $\hat{D}\left(\alpha_{\mathrm{S}}\right)$ admit Taylor expansions in $\alpha_{\mathrm{S}}$; the order at which the expansions are truncated defines the logarithmic accuracy of the resummation, as indicated in table 1 . In particular, to NNLL accuracy,

$$
\begin{aligned}
& A\left(\alpha_{\mathrm{S}}\right)=\frac{A_{1}}{4} \alpha_{\mathrm{S}}+\frac{A_{2}}{16} \alpha_{\mathrm{S}}^{2}+\frac{A_{3}}{64} \alpha_{\mathrm{S}}^{3} \\
& \hat{D}\left(\alpha_{\mathrm{S}}\right)=\hat{D}_{2} \alpha_{\mathrm{S}}^{2} .
\end{aligned}
$$

Note that there is an ambiguity in the way the expression eq. (2.4) is defined: specifically, one may rewrite eq. (2.4) as

$$
\begin{aligned}
C^{\mathrm{dQCD}}\left(N, m_{H}^{2}\right) & =g_{0}\left(\alpha_{\mathrm{S}}\left(m_{H}^{2}\right)\right) \exp S^{\mathrm{dQCD}}(\bar{\alpha} \ell, \bar{\alpha}) \\
\bar{\alpha} & \equiv 2 \beta_{0} \alpha_{\mathrm{S}}, \quad \ell \equiv \ln \frac{1}{N}
\end{aligned}
$$

where

$$
\begin{aligned}
g_{0}\left(\alpha_{\mathrm{S}}\right) & =1+\sum_{j=1}^{\infty} g_{0 j} \alpha_{\mathrm{S}}^{j} \\
S^{\mathrm{dQCD}}(\bar{\alpha} \ell, \bar{\alpha}) & =\frac{1}{\bar{\alpha}} g_{1}(\bar{\alpha} \ell)+g_{2}(\bar{\alpha} \ell)+\bar{\alpha} g_{3}(\bar{\alpha} \ell)+\bar{\alpha}^{2} g_{4}(\bar{\alpha} \ell)+\ldots
\end{aligned}
$$




\begin{tabular}{|lccccc|}
\hline & $(\mathrm{dQCD})$ & $A\left(\alpha_{\mathrm{S}}\right)$ & $\hat{D}\left(\alpha_{\mathrm{S}}\right)$ & $\hat{g}_{0}\left(\alpha_{\mathrm{S}}\right)$ & $\alpha_{\mathrm{S}}^{n} \ln ^{k} N$ \\
& $(\mathrm{SCET})$ & $\Gamma_{\text {cusp }}\left(\alpha_{\mathrm{S}}\right)$ & $\gamma_{W}\left(\alpha_{\mathrm{S}}\right)$ & $H, \tilde{s}_{\text {Higgs }}$ & $\alpha_{\mathrm{S}}^{n} \ln ^{k}\left(\mu_{s} / M\right)$ \\
\hline LL & & 1-loop & - & tree-level & $k=2 n$ \\
\hline NLL* & & 2-loop & 1-loop & tree-level & $2 n-1 \leq k \leq 2 n$ \\
NLL & & 2-loop & 1-loop & 1-loop & $2 n-2 \leq k \leq 2 n$ \\
\hline NNLL* & & 3-loop & 2-loop & 1-loop & $2 n-3 \leq k \leq 2 n$ \\
NNLL & & 3-loop & 2-loop & 2-loop & $2 n-4 \leq k \leq 2 n$ \\
\hline
\end{tabular}

Table 1. Orders of logarithmic approximations in resummed computations. The central three columns of the table give the order at which the functions which enter respectively the dQCD or SCET resummed expressions, as listed above the table, must be computed in order to achieve the accuracy called with the name given in the first column, and corresponding to the inclusion in the coefficient function of terms as given in the last column.

This differs from eq. (2.4) because now all constant (i.e., $N$-independent) terms are included in $g_{0}\left(\alpha_{\mathrm{S}}\right)$, while in eq. (2.4) some constant terms were exponentiated; of course, the difference is logarithmically subleading. In the sequel, we will use eq. (2.8) for numerical implementations.

We want to relate the dQCD expression eqs. (2.4)-(2.5) to its SCET counterpart. The latter, in the SCET approach of refs. [6, 8, 9, 19] is given directly in the space of the physical variable $z$, and it can be written as

$$
C^{\mathrm{SCET}}\left(z, m_{H}^{2}, \mu_{s}^{2}\right)=H\left(m_{H}^{2}\right) U\left(m_{H}^{2}, \mu_{s}^{2}\right) S\left(z, m_{H}^{2}, \mu_{s}^{2}\right),
$$

(the SCET result is actually more generally expressed as a function of other energy scales, which here we all take to be equal to the hard scale $m_{H}$ for simplicity, as this does not affect our arguments). The soft function is given by

$$
S\left(z, m_{H}^{2}, \mu_{s}^{2}\right)=\tilde{s}_{\text {Higgs }}\left(\ln \frac{m_{H}^{2}}{\mu_{s}^{2}}+\frac{\partial}{\partial \eta}, \mu_{s}\right) \frac{1}{1-z}\left(\frac{1-z}{\sqrt{z}}\right)^{2 \eta} \frac{e^{-2 \gamma_{E} \eta}}{\Gamma(2 \eta)}
$$

where

$$
\eta=\int_{m_{H}^{2}}^{\mu_{s}^{2}} \frac{d \mu^{2}}{\mu^{2}} A\left(\alpha_{\mathrm{S}}\left(\mu^{2}\right)\right)
$$

Finally,

$$
U\left(m_{H}^{2}, \mu_{s}^{2}\right)=\exp \int_{m_{H}^{2}}^{\mu_{s}^{2}} \frac{d \mu^{2}}{\mu^{2}}\left[A\left(\alpha_{\mathrm{S}}\left(\mu^{2}\right)\right) \ln \frac{m_{H}^{2}}{\mu^{2}}+\gamma_{W}\left(\alpha_{\mathrm{S}}\left(\mu^{2}\right)\right)\right]
$$

with $\gamma_{W}\left(\alpha_{\mathrm{S}}\right)=\gamma_{W, 2} \alpha_{\mathrm{S}}^{2}$ to NNLL accuracy. The hard function $H\left(m_{H}^{2}\right)$ admits an expansion in powers of $\alpha_{\mathrm{S}}\left(m_{H}^{2}\right)$, and $\tilde{s}_{\mathrm{Higgs}}\left(L, \mu_{s}^{2}\right)$ admits an expansion in powers of $\alpha_{\mathrm{S}}\left(\mu_{s}^{2}\right)$. The logarithmic accuracy of the resummation is fixed by the order at which these expansion are truncated, as shown in table 1 . Note that there is a slight ambiguity in the way the resummed expression eq. (2.12) is defined: in particular, once the various factor on the right-hand side are included to any desired order, the interference between them generates higher order terms: for example, if at NLL both $H$ and $\tilde{s}_{\text {Higgs }}$ are included up to order $\alpha_{\mathrm{S}}$, 

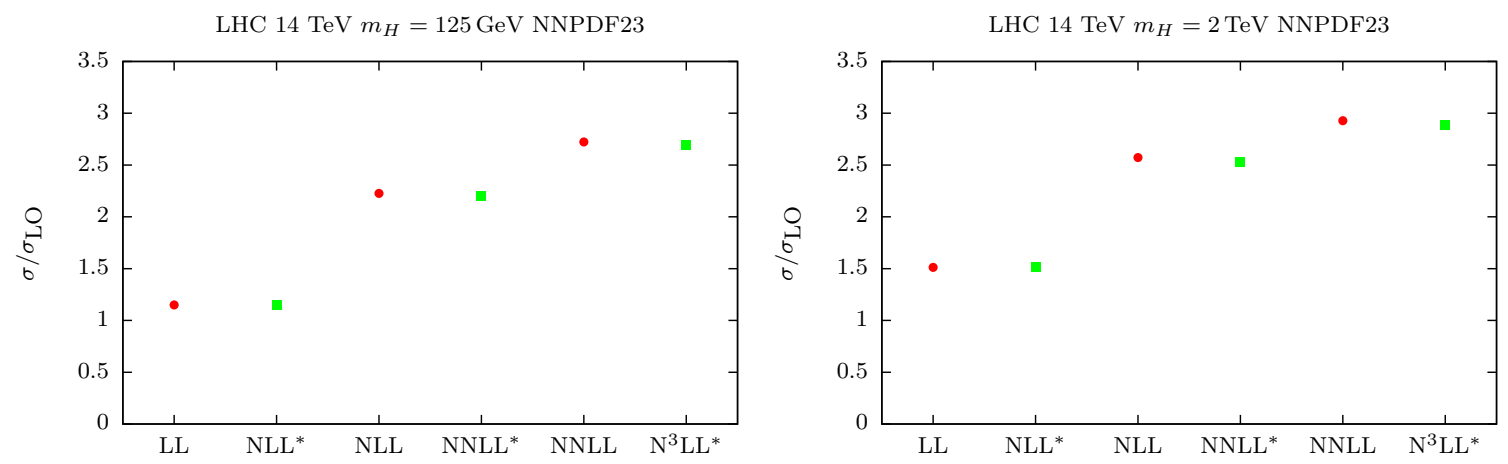

Figure 1. The cross-section for production of a Higgs-like particle of mass $m_{H}=125 \mathrm{GeV}$ (left) or $m_{H}=2 \mathrm{TeV}$ (right) in gluon fusion at the LHC $14 \mathrm{TeV}$ computed using the SCET expression at the various resummed orders of table 1. Results have been obtained using NNPDF2.3 NNLO PDFs with $\alpha_{s}\left(M_{Z}^{2}\right)=0.118$ and $\mu_{R}=\mu_{F}=m_{H}$.

their product will clearly include terms $\mathcal{O}\left(\alpha_{\mathrm{S}}^{2}\right)$. In this paper, specifically for all numerical implementations to be discussed in the sequel, we will consistently drop all higher-order interference terms, because this is the prescription used in the implementation of the results of refs. [5-7] in the RGHiggs code [32], as we have explicitly checked. This choice spoils the factorized form of the SCET result, eq. (2.12), though it simplifies the matching to fixed order.

Note that in refs. $[5,8,9,19]$ all results were presented in the starred (NLL*, NNLL* and so forth) approximation (and, somewhat confusingly, referred to as NLL and NNLL). The way the result of refs. $[5,8,9,19]$ could be upgraded to the more accurate NNLL approximation was presented in ref. [11] (SCET results corresponding to either starred or unstarred accuracy were considered e.g. in ref. [33] in the context of the resummation of jet vetoes).

For reference, in figure 1 we compare the total cross section for Higgs production in gluon fusion at the LHC $14 \mathrm{TeV}$, determined at the various resummed orders of table 1 , both using the starred result of refs. [5, 8, 9, 19] and its unstarred upgrade of ref. [11], for the physical value of the Higgs mass and for a very high-mass scalar, much closer to threshold (all other settings are the same used in all other plots in the sequel of the paper). While only the SCET result is shown in the plot, the dQCD result follows a similar pattern. It is clear that upgrading from the nonstarred to the starred result has a very substantial effect, while going up from the starred result at one order to the unstarred result at the subsequent order only has a very minor impact. Note also that it is natural to match the generic fixed order $\mathrm{N}^{k} \mathrm{LO}$ to either $\mathrm{N}^{k} \mathrm{LL}$ or $\mathrm{N}^{k+1} \mathrm{LL}{ }^{*}$, as done respectively in, e.g., ref. [2] and refs. [5, 8, 9, 19]; figure 1 shows that the extra effort needed in order to upgrade from $\mathrm{N}^{k} \mathrm{LL}$ to $\mathrm{N}^{k+1} \mathrm{LL}^{*}$ is unnecessary. Consequently we will henceforth only consider and compare the unstarred (NLL, NNLL etc.) results.

We now start from the dQCD expression eqs. (2.4)-(2.5). It is well known that $C^{\mathrm{dQCD}}\left(N, m_{H}^{2}\right)$, considered as a function of the complex variable $N$, has a branch cut on the positive real axis for $\bar{N} \geq N_{L}$, where $N_{L}$ is the position of the Landau pole of 
$\alpha_{\mathrm{S}}\left(m_{H}^{2} / \bar{N}^{2}\right)$

$$
1-2 \beta_{0} \alpha_{\mathrm{S}}\left(m_{H}^{2}\right) \ln N_{L}=0 .
$$

As a consequence, $\boldsymbol{C}^{\mathrm{dQCD}}\left(N, m_{H}^{2}\right)$ does not have an inverse Mellin transform. This is an effect of resummation: indeed, any finite-order truncation of the expansion of $\boldsymbol{C}^{\mathrm{dQCD}}\left(N, m_{H}^{2}\right)$ in powers of $\alpha_{\mathrm{S}}\left(m_{H}^{2}\right)$ does have a Mellin inverse [34].

We now show that a possible solution of the Landau pole problem, suggested by the structure of the SCET result, leads us directly to the SCET expression eq. (2.12) in a few simple steps. We first note that the exponent in the dQCD expression can be rewritten

$$
\hat{S}^{\mathrm{dQCD}}\left(m_{H}^{2}, \frac{m_{H}^{2}}{\bar{N}^{2}}\right)=\hat{S}^{\mathrm{dQCD}}\left(m_{H}^{2}, \mu_{s}^{2}\right)+\hat{S}^{\mathrm{dQCD}}\left(\mu_{s}^{2}, \frac{m_{H}^{2}}{\bar{N}^{2}}\right)
$$

where $\mu_{s}$ is an arbitrary energy scale. Now assume that we can choose $\mu_{s}$ so that $\alpha_{\mathrm{S}}\left(\mu_{s}^{2}\right)$ is in the perturbative domain, and at the same time

$$
\left|\ln \frac{\mu_{s}^{2}}{m_{H}^{2}}\right| \gg\left|\ln \frac{m_{H}^{2}}{\mu_{s}^{2} \bar{N}^{2}}\right|
$$

for all values of $N$ in the relevant range (whether such an energy scale actually exists, and how it can be determined in the context of dQCD, are issues that will be discussed in the next section). This means that $\mu_{s}$ is chosen to be of the same order of $\frac{m_{H}^{2}}{N^{2}}$, the scale which is being resummed in the dQCD approach.

In such case, the first term in eq. (2.17) contains the resummation, while the second term can be expanded in powers if $\alpha_{\mathrm{S}}\left(\mu_{s}^{2}\right)$ : it is on the same footing as the function $\hat{g}_{0}\left(\alpha_{\mathrm{S}}\right)$ of eq. (2.4), because now $\ln \frac{m_{H}^{2}}{\mu_{s}^{2} N^{2}}$ does not count as a large log (the large log is instead $\ln \frac{\mu_{s}^{2}}{m_{H}^{2}}$ ), and thus to NNLL (see table 1) it must be only kept up to $\mathcal{O}\left(\alpha_{\mathrm{S}}^{2}\right)$, neglecting $\mathcal{O}\left(\alpha_{\mathrm{S}}^{3}\right)$ terms. We find

$$
\begin{aligned}
\exp \hat{S}^{\mathrm{dQCD}}\left(\mu_{s}^{2}, \frac{m_{H}^{2}}{\bar{N}^{2}}\right) & =\exp \int_{\mu_{s}^{2}}^{\frac{m_{H}^{2}}{\bar{N}^{2}}} \frac{d \mu^{2}}{\mu^{2}}\left[A\left(\alpha_{\mathrm{S}}\left(\mu^{2}\right)\right) \ln \frac{m_{H}^{2}}{\mu^{2} \bar{N}^{2}}+\hat{D}\left(\alpha_{\mathrm{S}}\left(\mu^{2}\right)\right)\right] \\
& =F\left(L, \mu_{s}\right)
\end{aligned}
$$

where

$$
F\left(L, \mu_{s}\right)=1+\alpha_{\mathrm{S}}\left(\mu_{s}^{2}\right) \frac{A_{1} L^{2}}{8}+\alpha_{\mathrm{S}}^{2}\left(\mu_{s}^{2}\right)\left[\frac{A_{1}^{2} L^{4}}{128}-\frac{\beta_{0} A_{1} L^{3}}{24}+\frac{A_{2} L^{2}}{32}+\hat{D}_{2} L\right]+\mathcal{O}\left(\alpha_{\mathrm{S}}^{3}\right)
$$

and

$$
L=\ln \frac{m_{H}^{2}}{\mu_{s}^{2} \bar{N}^{2}} .
$$

Thus, we obtain a $\mu_{s}$-dependent version of the dQCD resummed cross section, which reads

$$
\begin{aligned}
C^{\mathrm{dQCD}}\left(N, m_{H}^{2}, \mu_{s}^{2}\right)= & \hat{g}_{0}\left(\alpha_{\mathrm{S}}\left(m_{H}^{2}\right)\right) F\left(L, \mu_{s}\right) \\
& \times \exp \int_{m_{H}^{2}}^{\mu_{s}^{2}} \frac{d \mu^{2}}{\mu^{2}}\left[A\left(\alpha_{\mathrm{S}}\left(\mu^{2}\right)\right) \ln \frac{m_{H}^{2}}{\mu^{2} \bar{N}^{2}}+\hat{D}\left(\alpha_{\mathrm{S}}\left(\mu^{2}\right)\right)\right] .
\end{aligned}
$$


Because we are keeping only a finite number of terms in the expansion of $F\left(L, \mu_{s}\right)$, the inverse Mellin transform is now well defined. We have thus arrived at a form of the dQCD result which is free of the Landau pole problem, in analogy to the SCET expression. Note that while up to NNLL $A\left(\alpha_{\mathrm{S}}\right)$ eq. (2.7) includes contributions up to $\mathcal{O}\left(\alpha_{\mathrm{S}}^{3}\right)$, only the $A_{1}$ and $A_{2}$ terms contribute to $F\left(L, \mu_{s}\right)$ eq. (2.20). This observation will help us in understanding the relation of the result found here with that of ref. [11].

We can now show that the form eq. (2.22) of the dQCD result coincides with the SCET result up to subleading terms. First, we note that the functions $F\left(L, \mu_{s}\right)$ and $\tilde{s}_{\text {Higgs }}\left(L, \mu_{s}\right)$ are closely related. Indeed, using the explicit expressions of the coefficients of the expansion of $\tilde{s}_{\text {Higgs }}\left(L, \mu_{s}\right)$ in powers of $\alpha_{\mathrm{S}}$,

$$
\tilde{s}_{\text {Higgs }}\left(L, \mu_{s}\right) \exp \left(-\frac{\zeta_{2} C_{A}}{2 \pi} \alpha_{\mathrm{S}}\left(\mu_{s}^{2}\right)\right)=F\left(L, \mu_{s}\right)+k \alpha_{\mathrm{S}}^{2}\left(\mu_{s}^{2}\right)+\mathcal{O}\left(\alpha_{\mathrm{S}}^{3}\right),
$$

where $k$ is a numerical constant. Recalling that $F\left(0, \mu_{s}\right)=1$, we find

$$
\frac{\tilde{s}_{\mathrm{Higgs}}\left(L, \mu_{s}\right)}{\tilde{s}_{\mathrm{Higgs}}\left(0, m_{H}\right)} \exp \left(-\frac{\zeta_{2} C_{A}}{2 \pi}\left(\alpha_{\mathrm{S}}\left(\mu_{s}^{2}\right)-\alpha_{\mathrm{S}}\left(m_{H}^{2}\right)\right)\right)=F\left(L, \mu_{s}\right)+\mathcal{O}\left(\alpha_{\mathrm{S}}^{3}\right)
$$

Also,

$$
\hat{g}_{0}\left(\alpha_{\mathrm{S}}\left(m_{H}^{2}\right)\right)=H\left(m_{H}^{2}\right) \tilde{s}\left(0, m_{H}\right)\left[1+\mathcal{O}\left(\alpha_{\mathrm{S}}^{3}\right)\right] .
$$

Finally, the first term in eq. (2.17) can be written as

$$
\begin{aligned}
\hat{S}^{\mathrm{dQCD}}\left(m_{H}^{2}, \mu_{s}^{2}\right) & =\int_{m_{H}^{2}}^{\mu_{s}^{2}} \frac{d \mu^{2}}{\mu^{2}}\left[A\left(\alpha_{\mathrm{S}}\left(\mu^{2}\right)\right) \ln \frac{m_{H}^{2}}{\mu^{2} \bar{N}^{2}}+\hat{D}\left(\alpha_{\mathrm{S}}\left(\mu^{2}\right)\right)\right] \\
& =\int_{m_{H}^{2}}^{\mu_{s}^{2}} \frac{d \mu^{2}}{\mu^{2}}\left[A\left(\alpha_{\mathrm{S}}\left(\mu^{2}\right)\right) \ln \frac{m_{H}^{2}}{\mu^{2}}+\hat{D}\left(\alpha_{\mathrm{S}}\left(\mu^{2}\right)\right)\right]+\ln \bar{N}^{-2 \eta},
\end{aligned}
$$

where $\eta$ is defined in eq. (2.14).

Substituting eqs. (2.24)-(2.26) in the Landau-pole free form of the dQCD result, eq. (2.22), we get

$$
\begin{aligned}
C^{\mathrm{dQCD}}\left(N, m_{H}^{2}, \mu_{s}^{2}\right)= & H\left(m_{H}^{2}\right) \tilde{s}_{\mathrm{Higgs}}\left(L, \mu_{s}\right) \bar{N}^{-2 \eta} \exp \left(\frac{\zeta_{2} C_{A}}{2 \pi}\left(\alpha_{\mathrm{S}}\left(m_{H}^{2}\right)-\alpha_{\mathrm{S}}\left(\mu_{s}^{2}\right)\right)\right) \\
& \times \exp \int_{m_{H}^{2}}^{\mu_{s}^{2}} \frac{d \mu^{2}}{\mu^{2}}\left[A\left(\alpha_{\mathrm{S}}\left(\mu^{2}\right)\right) \ln \frac{m_{H}^{2}}{\mu^{2}}+\hat{D}\left(\alpha_{\mathrm{S}}\left(\mu^{2}\right)\right)\right]\left(1+\mathcal{O}\left(\alpha_{\mathrm{S}}^{3}\right)\right) .
\end{aligned}
$$

This can be brought in the same form as the SCET result by noting that

$$
\int_{m_{H}^{2}}^{\mu_{s}^{2}} \frac{d \mu^{2}}{\mu^{2}} \hat{D}\left(\alpha_{\mathrm{S}}\left(\mu^{2}\right)\right)=\hat{D}_{2} \int_{m_{H}^{2}}^{\mu_{s}^{2}} \frac{d \mu^{2}}{\mu^{2}} \alpha_{\mathrm{S}}^{2}\left(\mu^{2}\right)=\frac{\hat{D}_{2}}{\beta_{0}}\left(\alpha_{\mathrm{S}}\left(m_{H}^{2}\right)-\alpha_{\mathrm{S}}\left(\mu_{s}^{2}\right)\right)
$$

to NNLL accuracy, and that

$$
\hat{D}_{2}+\frac{\zeta_{2} C_{A}}{2 \pi} \beta_{0}=\gamma_{W, 2}
$$


Hence

$$
\begin{aligned}
C^{\mathrm{dQCD}}( & \left.N, m_{H}^{2}, \mu_{s}^{2}\right) \\
& =H\left(m_{H}^{2}\right) \tilde{s}_{\mathrm{Higgs}}\left(L, \mu_{s}\right) \bar{N}^{-2 \eta} \exp \int_{m_{H}^{2}}^{\mu_{s}^{2}} \frac{d \mu^{2}}{\mu^{2}}\left[A\left(\alpha_{\mathrm{S}}\left(\mu^{2}\right)\right) \ln \frac{m_{H}^{2}}{\mu^{2}}+\gamma_{W}\left(\alpha_{\mathrm{S}}\left(\mu^{2}\right)\right)\right] \\
& =H\left(m_{H}^{2}\right) U\left(m_{H}^{2}, \mu_{s}^{2}\right) \tilde{s}_{\mathrm{Higgs}}\left(L, \mu_{s}\right) \bar{N}^{-2 \eta}\left(1+\mathcal{O}\left(\alpha_{\mathrm{S}}^{3}\right)\right),
\end{aligned}
$$

which is the Landau-pole-free form of the dQCD result, written in SCET factorized form. For future convenience, we define the ratio of the starting dQCD expression eq. (2.4) to this intermediate result (eq. (2.30)):

$$
\boldsymbol{C}_{r}^{(1)}\left(N, m_{H}^{2}, \mu_{s}^{2}\right) \equiv \frac{\boldsymbol{C}^{\mathrm{dQCD}}\left(N, m_{H}^{2}\right)}{H\left(m_{H}^{2}\right) U\left(m_{H}^{2}, \mu_{s}^{2}\right) \tilde{s}_{\mathrm{Higgs}}\left(L, \mu_{s}\right) \bar{N}^{-2 \eta}} .
$$

It is now immediate to show that the result eq. (2.30) coincides with the Mellin transform of the SCET coefficient function, up to power-suppressed terms. Indeed,

$$
\boldsymbol{C}^{\mathrm{SCET}}\left(N, m_{H}^{2}, \mu_{s}^{2}\right)=H\left(m_{H}^{2}\right) U\left(m_{H}^{2}, \mu_{s}^{2}\right) \boldsymbol{S}\left(N, m_{H}^{2}, \mu_{s}^{2}\right),
$$

where

$$
\boldsymbol{S}\left(N, m_{H}^{2}, \mu_{s}^{2}\right)=\tilde{s}_{\mathrm{Higgs}}\left(\ln \frac{m_{H}^{2}}{\mu_{s}^{2}}+\frac{\partial}{\partial \eta}, \mu_{s}\right) \frac{\Gamma(N-\eta)}{\Gamma(N+\eta)} e^{-2 \gamma_{E} \eta}
$$

for fixed (i.e., $z$-independent) $\mu_{s}$. But up to power-suppressed terms

$$
\begin{aligned}
\boldsymbol{S}\left(N, m_{H}^{2}, \mu_{s}^{2}\right) & =\tilde{s}_{\text {Higgs }}\left(\ln \frac{m_{H}^{2}}{\mu_{s}^{2}}+\frac{\partial}{\partial \eta}, \mu_{s}\right) \bar{N}^{-2 \eta}+\mathcal{O}\left(\frac{1}{N}\right) \\
& =\tilde{s}_{\text {Higgs }}\left(\ln \frac{m_{H}^{2}}{\mu_{s}^{2} \bar{N}^{2}}, \mu_{s}\right) \bar{N}^{-2 \eta}\left(1+\mathcal{O}\left(\frac{1}{N}\right)\right) .
\end{aligned}
$$

Thus, up to terms suppressed in the large- $N$ limit,

$$
C^{\mathrm{SCET}}\left(N, m_{H}^{2}, \mu_{s}^{2}\right)=H\left(m_{H}^{2}\right) U\left(m_{H}^{2}, \mu_{s}^{2}\right) \tilde{s}_{\mathrm{Higgs}}\left(L, \mu_{s}\right) \bar{N}^{-2 \eta}\left(1+\mathcal{O}\left(\frac{1}{N}\right)\right),
$$

which is what we set out to prove. We also define the ratio of the starting dQCD expression eq. (2.4) to the exact Mellin transform eq. (2.32) of the SCET expression eq. (2.12):

$$
\boldsymbol{C}_{r}^{(2)}\left(N, m_{H}^{2}, \mu_{s}^{2}\right)=\frac{\boldsymbol{C}^{\mathrm{dQCD}}\left(N, m_{H}^{2}\right)}{\boldsymbol{C}^{\mathrm{SCET}}\left(N, m_{H}^{2}, \mu_{s}^{2}\right)} .
$$

The steps leading from the Landau-pole-free dQCD expression eq. (2.30) to the standard SCET expression eq. (2.12) can also be traced in $z$ space. Indeed, the $z$-space form of the expression eq. (2.30) is obtained by inverse Mellin transformation, and it is given by

$$
S\left(z, M^{2}, \mu_{s}^{2}\right)=\tilde{s}_{\text {Higgs }}\left(\ln \frac{M^{2}}{\mu_{s}^{2}}+\frac{\partial}{\partial \eta}, \mu_{s}\right)(-\ln z)^{-1+2 \eta} \frac{e^{-2 \gamma_{E} \eta}}{\Gamma(2 \eta)} .
$$


Noting that

$$
(-\ln z)^{-1+2 \eta}=\frac{1}{1-z}\left(\frac{1-z}{\sqrt{z}}\right)^{2 \eta}[1+\mathcal{O}(1-z)]
$$

it follows that eq. (2.37) differs from the starting SCET expression of the soft function eq. (2.13) by terms which are suppressed by positive powers of $1-z$. It is interesting to observe that

$$
(-\ln z)^{-1+2 \eta}=\frac{\sqrt{z}}{1-z}\left(\frac{1-z}{\sqrt{z}}\right)^{2 \eta}\left[1+\mathcal{O}\left((1-z)^{2}\right)\right]
$$

i.e., the leading power-suppressed difference can be in fact expressed as a $\sqrt{z}$ prefactor, with further power suppressed differences only arising at $\mathcal{O}\left((1-z)^{2}\right)$. It was pointed out in ref. [3] (see in particular the discussion of eq. (2.43) of this reference) that this $\sqrt{z}$ prefactor has a non-negligible impact. While the reader is referred to refs. [3, 30] for a discussion of the motivation and quantitative impact of this factor, we recall here that its effect goes in the same direction as the so-called collinear improvement of the resummed result, which extends the accuracy of the resummed results to power suppressed terms (at the leading logarithmic level). As a consequence, the SCET result is the unimproved result, while the dQCD result is closer to the collinear-improved result. We will come back to an assessment of the impact of this term in the context of the SCET vs. dQCD comparison in section 4 below.

We have thus shown that the dQCD result coincides with the SCET result in two steps. In the first step, we have changed the resummed scale from $\frac{m_{H}^{2}}{N^{2}}$ to $\mu_{s}^{2}$. If $\ln \frac{m_{H}^{2}}{\mu_{s}^{2} N^{2}}$ is not a large log, this leads to the intermediate result eq. (2.30), which only differs from the starting dQCD expression by a factor $\boldsymbol{C}_{r}^{(1)}\left(N, m_{H}^{2}, \mu_{s}^{2}\right)$, that only contains subleading logarithmic terms, generated by the interference of the neglected $\mathcal{O}\left(\alpha_{\mathrm{S}}^{3}\right)$ terms with the logs which are being resummed. In the second step, the result eq. (2.30) is found to coincide with the exact Mellin transform of the SCET expression eq. (2.33), up to power-suppressed terms: hence the SCET and QCD expressions differ by a combined factor $\boldsymbol{C}_{r}^{(2)}\left(N, m_{H}^{2}, \mu_{s}^{2}\right)$ which contains both logarithmically subleading and power-suppressed terms.

We conclude this section by briefly discussing the relation of the result we just obtained to the "master formula" relating dQCD and SCET given in ref. [11]. In that reference, we started with the SCET expression, and we rewrote it by freely modifying subleading terms (both log and power suppressed). We ended up with the expression of eq. (2.19), but with only the $A_{1}$ and $A_{2}$ contributions to $A\left(\alpha_{\mathrm{S}}\right)$ included in $S^{\mathrm{dQCD}}\left(\mu_{s}^{2}, \frac{m_{H}^{2}}{N^{2}}\right)$. Because however $S^{\mathrm{dQCD}}\left(m_{H}^{2}, \mu_{s}^{2}\right)$ also includes the $A_{3}$ term, if one substitutes eq. (2.17) with the two terms on the right-hand side computed thus in the expression eq. (2.4) of the dQCD coefficient, one gets a result which differs from the starting dQCD coefficient function by a multiplicative factor

$$
\boldsymbol{C}_{r}^{(0)}\left(N, m_{H}^{2}, \mu_{s}^{2}\right)=\exp \int_{\mu_{s}^{2}}^{\frac{m_{H}^{2}}{\bar{N}^{2}}} \frac{d \mu^{2}}{\mu^{2}} \ln \frac{m_{H}^{2}}{\mu^{2} \bar{N}^{2}}\left[A\left(\alpha_{\mathrm{S}}\left(\mu^{2}\right)\right)-A_{1} \alpha_{\mathrm{S}}\left(\mu^{2}\right)-A_{2} \alpha_{\mathrm{S}}^{2}\left(\mu^{2}\right)\right] .
$$

This is the result of ref. [11] (where $\boldsymbol{C}_{r}^{(0)}\left(N, m_{H}^{2}, \mu_{s}^{2}\right)$ was called $C_{r}$ ), which thus corresponds to the very first step of the derivation presented here. 


\section{Factorization and saddle-point approximation}

The main result of the previous section is that the Landau pole can be removed from the dQCD expression, at the cost of introducing the dependence on an extra scale $\mu_{s}$. The ensuing expression eq. (2.22) only differs from the SCET result by logarithmically subleading and power-suppressed terms.

However, one must then make a choice for the scale $\mu_{s}$, such that the Landau pole is avoided. If, for instance, one chooses $\mu_{s}$ proportional to the partonic scale $m_{H}(1-z)$, with $z$ the parton momentum fraction, (a similar choice is made in ref. [35] for threshold resummation of the top production cross-section) then the Landau pole reappears when integrating over $z$. In refs. $[5,8,9,19]$ it was suggested that one may choose $\mu_{s}$ to be proportional to a hadronic scale:

$$
\mu_{s}=m_{H}(1-\tau) g(\tau)
$$

where the function $g(\tau)$ is fixed in two different ways, both of which attempt to minimize the contribution to the cross-section coming from the one-loop term in $\tilde{s}_{\text {Higgs }}$ eq. (2.13). As discussed in section 2, this choice is justified to the extent that the condition eq. (2.18) is satisfied. Henceforth, unless otherwise stated, SCET results will be presented by taking for $\mu_{s}$ the average of these scale choices. A discussion of scale choices will then be provided in the end section 4 (in particular figure 5) below.

With this choice, the problem of the Landau pole does not arise; however, the partonic cross-section depends on a hadronic scale, and thus, because $C$ depends on $\tau$, the hadronic cross-section eq. (2.1) no longer has the form of a convolution. In particular, this means that the hadronic cross-section no longer factorizes upon taking a Mellin transform eq. (2.2) - we will refer to this as breaking of Mellin factorization, or factorization breaking, for short.

A priori, this appears to prevent a direct comparison of the SCET and dQCD expressions at the level of partonic cross-sections: the comparison can only be done at the hadronic level. Indeed, the scale whose logs are being resummed in eq. (2.22) is $\mu_{s}$; it follows that, as already pointed out in ref. [10], the equivalence of the dQCD and SCET resummation only holds if no further logs are generated by the convolution integral, which in turn depends on the form of the parton distributions. Quite apart from this issue of principle, there remains the practical issue that, apparently, a comparison of resummed expressions obtained with this choice of scale to the standard factorized expressions, is only possible once a particular PDF has been chosen.

We will now show that this difficulty can be circumvented by using a saddle-point method. Before doing this, we dispose of a technical difficulty. As mentioned in the previous section, because of the Landau pole, $\boldsymbol{C}^{\mathrm{dQCD}}\left(N, m_{H}^{2}\right)$ eq. (2.4) does not have a Mellin inverse; however, any finite-order truncation of it does. There are several ways of dealing with this problem which (unlike the method of the previous section) do not require introducing an extra scale. They all amount to expanding the resummed result in powers of $\alpha_{\mathrm{S}}$, performing the Mellin inversion term by term, and viewing the divergent series which is obtained as an asymptotic series. As shown in ref. [34], one has to go 
extremely close to the Landau pole for this to make any difference: it is only for $\tau \gtrsim 0.9$ that resummed results obtained summing the asymptotic series in different ways differ by a significant amount. Hence, for the sake of the discussion in the present paper this point is immaterial, especially since we mostly deal with processes for which the resummed series is perturbative, i.e. $\alpha_{\mathrm{S}} \ln ^{2}(1-\tau) \ll 1$.

In all the subsequent discussion the Mellin inversion integral

$$
\sigma^{\mathrm{dQCD}}\left(\tau, m_{H}^{2}\right)=\frac{1}{2 \pi i} \int_{c-i \infty}^{c+i \infty} d N \tau^{-N} C^{\mathrm{dQCD}}\left(N, m_{H}^{2}\right) \mathcal{L}(N)
$$

can be thought of as being performed while taking for $\boldsymbol{C}^{\mathrm{dQCD}}\left(N, m_{H}^{2}\right)$ any sufficiently highorder truncation of the perturbative expansion. In practice, the integrals will be computed by choosing a path which intercepts the real axis to the left of the Landau pole, but to the right of of all other singularities of the Mellin transform (this is the so-called minimal prescription [18]).

We now explain how using the saddle-point method we can compare results obtained using the scale choice eq. (3.1) with standard factorized results at the level of partonic cross-sections: the idea is that the saddle-point approximation gives a way of directly relating hadronic and partonic kinematics $[29,36]$. In the saddle-point approximation, the Mellin inversion integral eq. (3.2) is given by

$$
\sigma^{\mathrm{dQCD}}\left(\tau, m_{H}^{2}\right) \approx Z^{\mathrm{dQCD}} \tau^{-N_{0}^{\mathrm{dQCD}}(\tau)} \mathcal{L}\left(N_{0}^{\mathrm{dQCD}}(\tau)\right) \boldsymbol{C}^{\mathrm{dQCD}}\left(N_{0}^{\mathrm{dQCD}}(\tau), m_{H}^{2}\right)
$$

where the saddle point $N_{0}^{\mathrm{dQCD}}(\tau)$ is found by solving for the condition

$$
\left.\frac{d}{d N} E_{\mathrm{dQCD}}\left(\tau, N ; m_{H}^{2}\right)\right|_{N=N_{0}^{\mathrm{dQCD}}}=0
$$

where

$$
E_{\mathrm{dQCD}}\left(\tau, N ; m_{H}^{2}\right)=N \ln \frac{1}{\tau}+\ln \boldsymbol{C}^{\mathrm{dQCD}}\left(N, m_{H}^{2}\right)+\ln \mathcal{L}(N)
$$

and $Z^{\mathrm{dQCD}}$ is a fluctuation term given by

$$
Z^{\mathrm{dQCD}}=\frac{1}{\sqrt{2 \pi E_{\mathrm{dQCD}}^{\prime \prime}\left(\tau, N_{0}^{\mathrm{dQCD}}(\tau) ; m_{H}^{2}\right)}} .
$$

The main reason why the saddle-point expression eq. (3.3) is interesting is that it turns the convolution in the $\tau$-space expression eq. (2.1) into an ordinary product, if the $\tau$-space expression is viewed as an inverse Mellin transform, with the Mellin inversion integral evaluated by saddle point. Indeed, each factor in eq. (3.3) is a function of $\tau$ through $N_{0}^{\mathrm{dQCD}}(\tau)$. A further simplification comes from the fact that in practice the subasymptotic fluctuation term $Z^{\mathrm{dQCD}}$ eq. (3.6) is dominated by the parton luminosity, so that it is essentially independent of the coefficient function.

This provides us with an elegant solution to the problem of comparing momentumspace hadron-level cross-sections in a way which separates the effect of the parton luminosity and of the coefficient function. Indeed, consider the case of the SCET resummed 
coefficient function eq. (2.12). If one uses such an expression in the expression eq. (2.1) of the hadronic cross section, it is still true that

$$
\boldsymbol{\sigma}\left(N, m_{H}^{2}, \mu_{s}^{2}\right)=C^{\mathrm{SCET}}\left(N, m_{H}^{2}, \mu_{s}^{2}\right) \mathcal{L}(N)
$$

provided only the Mellin transform is performed at fixed $\mu_{s}$. Of course, if $\mu_{s}$ depends on $\tau$, then upon Mellin inversion $\boldsymbol{\sigma}\left(N, m_{H}^{2}, \mu_{s}^{2}\right)$ is not the full Mellin transform of $\boldsymbol{\sigma}\left(\tau, m_{H}^{2}, \mu_{s}\right)$, in that the $\tau$-dependence through $\mu_{s}$ is not transformed.

Be that as it may, it is still true that

$$
\sigma^{\mathrm{SCET}}\left(\tau, m_{H}^{2}, \mu_{s}^{2}\right) \approx Z^{\mathrm{SCET}} \tau^{-N_{0}^{\mathrm{SCET}}(\tau)} \mathcal{L}\left(N_{0}^{\mathrm{SCET}}(\tau), m_{H}^{2}\right) C^{\mathrm{SCET}}\left(N_{0}^{\mathrm{SCET}}(\tau), m_{H}^{2}, \mu_{s}^{2}\right)
$$

where now

$$
E_{\mathrm{SCET}}\left(\tau, N ; m_{H}^{2}\right)=N \ln \frac{1}{\tau}+\ln C^{\mathrm{SCET}}\left(N, m_{H}^{2}\right)+\ln \mathcal{L}(N),
$$

and the position of the saddle point and the fluctuation term are determined in analogy to eqs. (3.4), (3.6).

Therefore, when comparing the $\tau$-space dQCD and SCET expressions, we can separate the effect of the PDF from that of the coefficient function. The former is entirely due to the fact that, in general, the position of the saddle point is different, so that $N_{0}^{\mathrm{SCET}} \neq N_{0}^{\mathrm{dQCD}}$, and thus the PDF-dependent factors $\mathcal{L}$ and $Z$ do not cancel in the ratio of $\sigma^{\mathrm{SCET}}\left(\tau, m_{H}^{2}, \mu_{s}^{2}\right)$ to $\sigma^{\mathrm{dQCD}}\left(\tau, m_{H}^{2}\right)$ because they are evaluated at different values of $N_{0}(\tau)$. The latter, instead, is due to the fact that even at the same value of $N, C^{\mathrm{dQCD}}$ and $\boldsymbol{C}^{\mathrm{SCET}}$ do not coincide. The violation of Mellin factorization now manifests itself as the fact that, while $N_{0}^{\mathrm{dQCD}}$ only depends on $\tau$ through the solution of the saddle point condition eq. (3.4), $N_{0}^{\mathrm{SCET}}$ has a further, explicit $\tau$ dependence, due to the fact that $\mu_{s}$ now also depends on $\tau$.

We can now use this method to compare the dQCD resummation to the SCET resummation with the scale choice eq. (3.1). Here and henceforth all dQCD results are obtained using a variant of the ResHiggs code [37] of ref. [38]; SCET results are obtained using our own computer implementation, which has been cross-checked and benchmarked against the RGHiggs code [32] of refs. [5-7]. First, we check the accuracy of the saddle approximation. In figure 2 we compare the resummed coefficient function in dQCD and SCET to its saddle approximation, for a wide range of Higgs masses. In the case of SCET, $\mu_{s}$ is chosen as suggested in refs. $[6,9,19]$, i.e. according to eq. (3.1) and with the function $g(\tau)$ as given there (see section 4 and specifically figure 4 below for a more explicit discussion). Here and in the sequel we will always use NNLO NNPDF2.3 PDFs [39], with $\alpha_{\mathrm{S}}\left(M_{Z}^{2}\right)=0.118$ and a maximum $n_{f}=5$ flavor scheme. Note that, as mentioned in the introduction, here and henceforth we will always consider a range of value of $m_{H}$ that goes well beyond the acceptable physical Higgs mass region, in order to see the qualitative behaviour also in high-mass regions closer to threshold, which may be of relevance for other processes, such as Drell-Yan production. It is clear that the relative deviation between the exact result and the saddle approximation is always below $2 \%$. In fact, note that the deviation goes in the same direction for the SCET and dQCD results, so using the saddle approximation to evaluate their ratio is yet more accurate. 


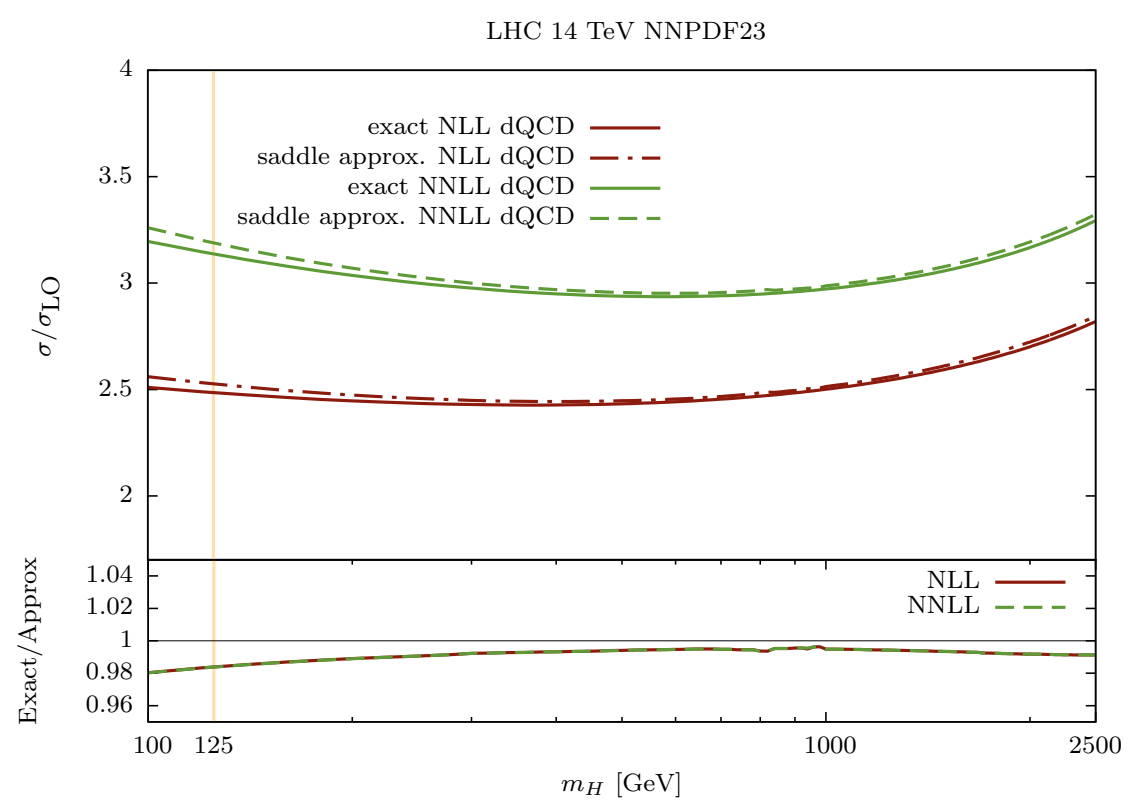

LHC 14 TeV NNPDF23

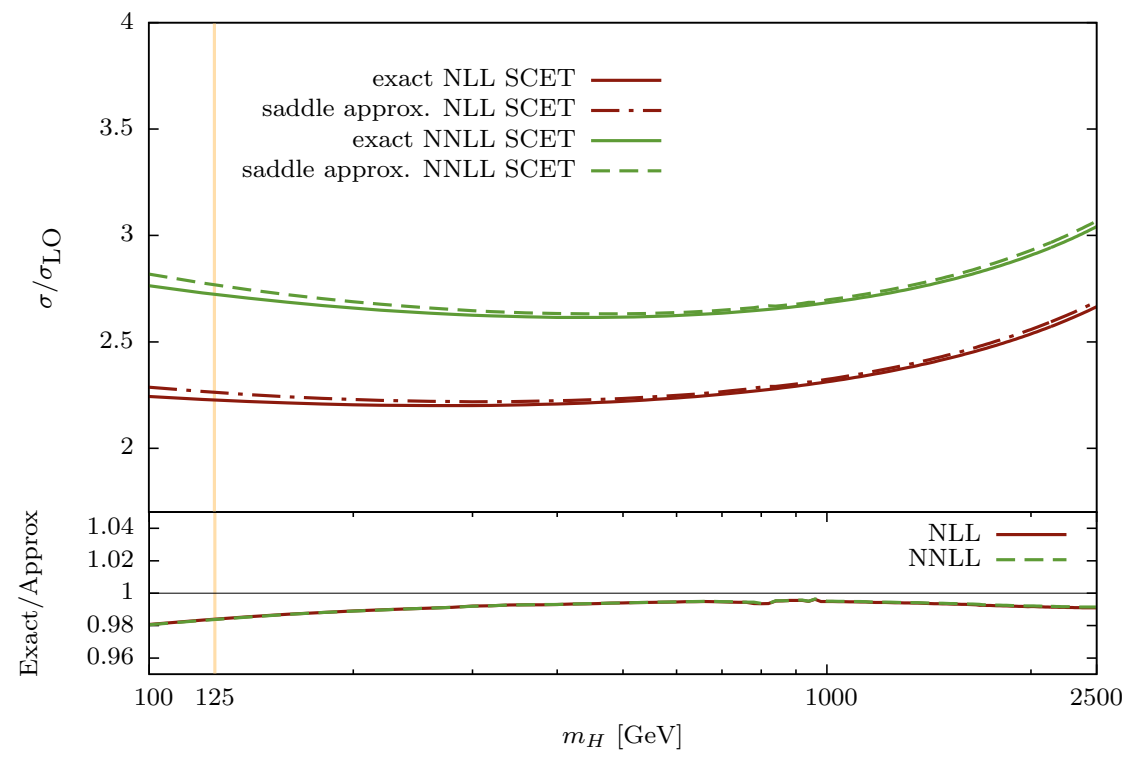

Figure 2. Comparison of the saddle-point approximation (dashed curves) to the exact result (solid curves) for the ratio of the resummed to leading-order cross section for Higgs in gluon fusion at NLL (bottom curves, green) and NNLL (top curves, red), computed using dQCD (top plot) and SCET (bottom plot), at LHC $\sqrt{s}=14 \mathrm{TeV}$, for a wide (unphysical) range of the "Higgs" mass. We use NNPDF2.3 PDFs with $\alpha_{\mathrm{S}}\left(M_{Z}^{2}\right)=0.118$.

Having established the accuracy of the saddle-point approximation, we proceed to estimating the violation of Mellin factorization, which, as mentioned, can only manifest itself in the position of the saddle being different for dQCD and SCET, $N_{0}^{\mathrm{SCET}} \neq N_{0}^{\mathrm{dQCD}}$. The position of the saddle is determined through the condition eq. (3.4) by the explicit form of the coefficient function: hence, even a pair of coefficient functions which do respect 


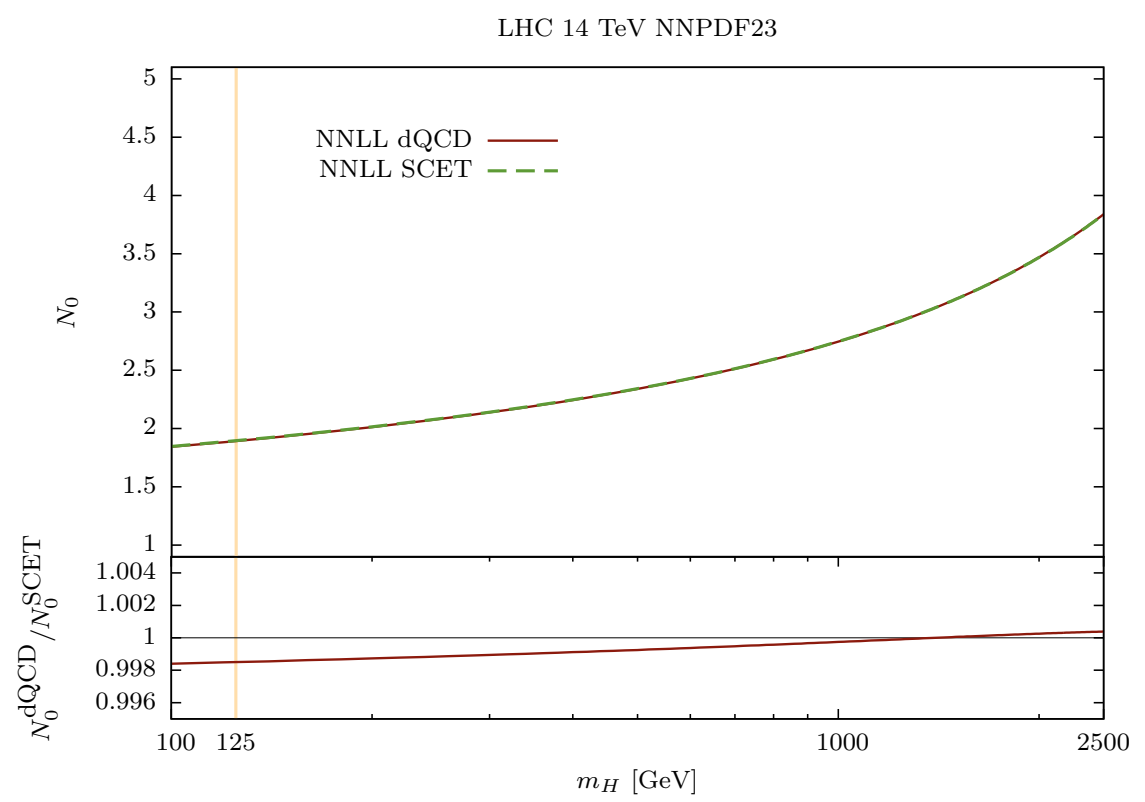

Figure 3. The saddle points $N_{0}^{\mathrm{dQCD}}$ (solid curve) and $N_{0}^{\mathrm{SCET}}$ (dashed) computed using respectively eq. (3.5) and eq. (3.9) in eq. (3.4) (top); their ratio is also shown (bottom). All settings are the same as in figure 2.

factorization, but differ in any aspect (such as the way the resummation is implemented) will generally lead to different saddle-points values. Therefore the deviation from unity of the ratio $\frac{N_{0}^{\mathrm{dQCD}}}{N_{0}^{\mathrm{SCET}}}$ provides an upper bound to the violation of factorization: the shift in the position of the saddle measures the maximal effect of factorization violation.

In figure 3 we display $N_{0}^{\mathrm{dQCD}}, N_{0}^{\mathrm{SCET}}$ and their ratio, for the same kinematics and with the same settings as for figure 2. It is clear that the difference is at the permille level in the whole range. We conclude that, in practice, the effect of the violation of Mellin factorization is negligible. We understand this as a consequence of the fact that the position of the saddle point is dominated by the parton luminosity, i.e. the contribution of $\boldsymbol{C}^{\mathrm{dQCD}}\left(N, m_{H}^{2}\right)$ and $\boldsymbol{C}^{\mathrm{SCET}}\left(N, m_{H}^{2}\right)$ to eqs. (3.5) and (3.9) respectively is completely negligible, as already observed in ref. [29] (see in particular figure 3). We conclude that in practice $N_{0}^{\mathrm{dQCD}} \approx N_{0}^{\mathrm{SCET}}$ to very good approximation.

It then immediately follows from eq. (3.3) that

$$
\frac{\sigma^{\mathrm{dQCD}}\left(\tau, m_{H}^{2}\right)}{\sigma^{\mathrm{SCET}}\left(\tau, m_{H}^{2}, \mu_{s}^{2}(\tau)\right)} \approx \frac{C^{\mathrm{dQCD}}\left(N_{0}^{\mathrm{dQCD}}(\tau), m_{H}^{2}\right)}{C^{\mathrm{SCET}}\left(N_{0}^{\mathrm{SCET}}(\tau), m_{H}^{2}, \mu_{s}^{2}(\tau)\right)}=C_{r}^{(2)}\left(N_{0}(\tau), m_{H}^{2}, \mu_{s}^{2}(\tau)\right),
$$

where the first approximate equality follows from the fact that $N_{0}^{\mathrm{dQCD}} \approx N_{0}^{\mathrm{SCET}}=N_{0}$ and $Z^{\mathrm{dQCD}} \approx Z^{\mathrm{SCET}}$, in the last step we have used the definition eq. (2.36), and the only remaining trace of violation of factorization is in the the dependence of $\mu_{s}$ on $\tau$, which we have explicitly indicated. We have checked that $\boldsymbol{C}_{r}^{(2)}\left(N_{0}(\tau), m_{H}^{2}, \mu_{s}^{2}\right)$ coincides with the ratio of physical cross-sections according to eq. (3.10), up to corrections which never exceed $0.4 \%$ in the region shown, in agreement with the conclusion reached from figure 3 . 
We conclude that with this scale choice the violation of Mellin factorization in the SCET expression has a negligible impact, and that further differences between the SCET and dQCD results in $\tau$ space and at the hadronic level can be reduced to parton-level results in $N$-space, and in particular can be assessed by studying the three ratio functions $\boldsymbol{C}_{r}^{(i)}\left(N, m_{H}^{2}, \mu_{s}^{2}\right)$ eqs. $(2.31),(2.36),(2.40)$, all evaluated at $N=N_{0}(\tau)$.

Before concluding this section, we note that the saddle-point approach allows us to better understand and generalize a result of ref. [12]. There, it was argued that, if the luminosity behaves as

$$
\mathcal{L}(z) \sim z^{-s_{1}}, \quad s_{1}>0
$$

in the relevant range of integration, as assumed for example in ref. [9], then

$$
\sigma\left(\tau, m_{H}^{2}\right)=\int_{\tau}^{1} \frac{d z}{z} \mathcal{L}\left(\frac{\tau}{z}\right) C\left(z, m_{H}^{2}\right)=\mathcal{L}(\tau) \int_{\tau}^{1} \frac{d z}{z} z^{s_{1}} C\left(z, m_{H}^{2}\right)
$$

which is just the $s_{1}^{\text {th }}$ Mellin moment of $C\left(z, m_{H}^{2}\right)$ times the luminosity, up to corrections of order $\mathcal{O}\left(\tau^{s_{1}}\right)$, which is negligible for $\tau \ll 1$. The same result can be obtained through the saddle-point approximation. Indeed, assuming eq. (3.11), we have

$$
\sigma\left(\tau, m_{H}^{2}\right)=\frac{1}{2 \pi i} \int_{c-i \infty}^{c+i \infty} d N \tau^{-N} \frac{1}{N-s_{1}} \boldsymbol{C}\left(N, m_{H}^{2}\right) .
$$

It is easy to check that, for $\tau \ll 1$, the coefficient function does not affect the position of the saddle point, which is given by

$$
N_{0} \simeq s_{1}+\frac{1}{\ln \frac{1}{\tau}}
$$

and the saddle-point approximation of the inversion integral gives

$$
\sigma\left(\tau, m_{H}^{2}\right) \approx \frac{e}{\sqrt{2 \pi}} \tau^{-s_{1}} \boldsymbol{C}\left(N_{0}, m_{H}^{2}\right) \simeq \frac{e}{\sqrt{2 \pi}} \mathcal{L}(\tau) \boldsymbol{C}\left(s_{1}, m_{H}^{2}\right)
$$

where in the last step we have neglected corrections of order $1 /|\ln \tau|$ to $\boldsymbol{C}\left(s_{1}, m_{H}^{2}\right)$, as appropriate for $\tau \ll 1$. This is the same result obtained in ref. [12], since $e / \sqrt{2 \pi} \simeq 1.084$.

\section{Logarithmically subleading vs. power-suppressed differences: an as- sessment}

Having established that, through the saddle-point method, SCET and dQCD cross sections can be compared at the partonic level, we now assess the quantitative impact of the differences that were presented analytically in section 2 .

In that section, we saw that we can obtain the SCET expression from the dQCD one in two steps: first, by introducing the dependence on the scale $\mu_{s}$ and expanding in order to get rid of the Landau pole, which leads to an expression which differs from the starting dQCD one by logarithmically subleading terms. In $N$ space, the ratio of these two expression is given by $\boldsymbol{C}_{r}^{(1)}\left(N, m_{H}^{2}, \mu_{s}^{2}\right)$ eq. (2.31). In the second step, powersuppressed terms are introduced, in order to get the standard SCET expression, whose 

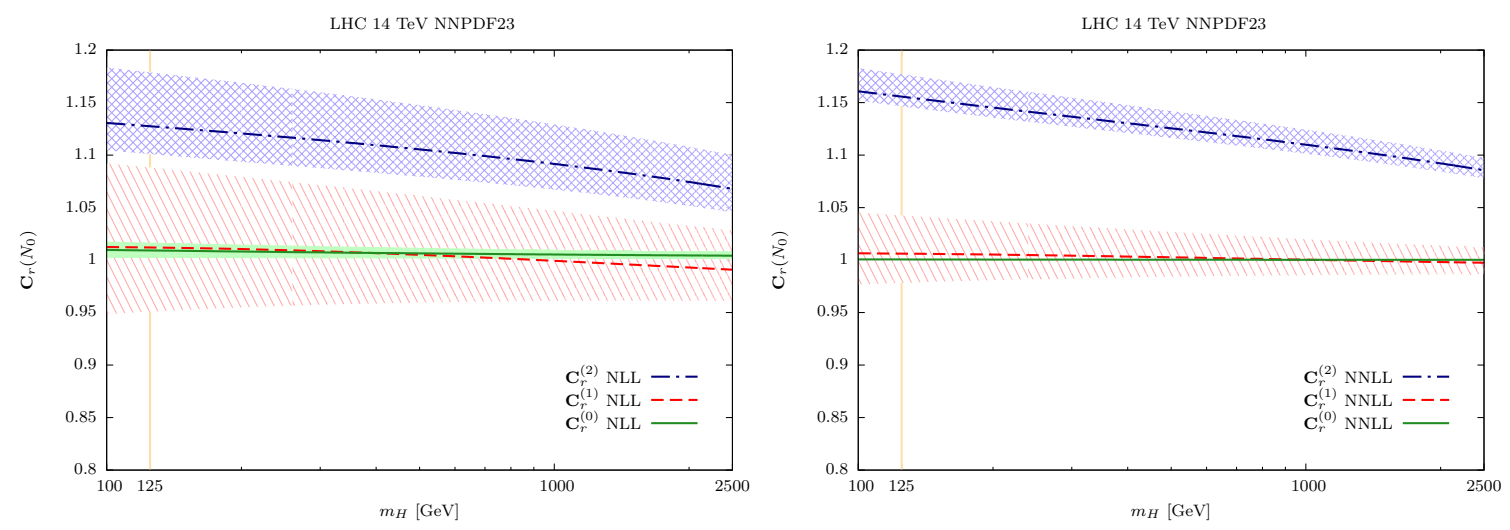

Figure 4. The ratio functions $\boldsymbol{C}_{r}^{(i)}\left(N_{0}(\tau), M^{2}, \mu_{s}^{2}\right)$ eqs. (2.31), (2.36), (2.40), computed at NLL (left) and NNLL (right) as a function of the saddle point $N_{0}(\tau)$, and plotted versus the Higgs mass $m_{H}$. All settings are the same as in figure 2. The curves shown are, from top to bottom, $\boldsymbol{C}_{r}^{(2)}$, $\boldsymbol{C}_{r}^{(0)}$, and $\boldsymbol{C}_{r}^{(1)}$. In each case, the edges of the band correspond to the two different choices for $\mu_{s}$ from refs. $[6,9,19]$, and the central curve is their average.

ratio to the starting dQCD one is $\boldsymbol{C}_{r}^{(2)}\left(N, m_{H}^{2}, \mu_{s}^{2}\right)$ eq. (2.36). A preliminary step, which corresponds to the "master formula" of ref. [11], corresponds to comparing results after introducing the dependence on $\mu_{s}$ in the dQCD result, but before expanding it out, which may already introduce some subleading $\mu_{s}$-dependent terms, whose ratio to the starting QCD expression is given by $\boldsymbol{C}_{r}^{(0)}\left(N, m_{H}^{2}, \mu_{s}^{2}\right)$ eq. (2.40). In $z$ space, in the first step the starting dQCD expression is turned into an expression which has the same form as the SCET result, but with the soft function eq. (2.13) replaced by its large- $z$ form eq. (2.37). In the second step, one ends up with the standard resummed SCET result.

In figure 4, we plot the three ratio functions $C_{r}^{(i)}\left(N, m_{H}^{2}, \mu_{s}^{2}\right)$, all evaluated (at the NLL and NNLL level) at $N=N_{0}(\tau)$, as functions of $m_{H}$. In each case, we show results as a band whose edges are obtained using $\mu_{s}$ eq. (3.1) computed using either of the two choices for $g(\tau)$ from refs. [6, 9, 19], with a central curve obtained using the average of these two scale choices; of course, the dependence on the choice of $\mu_{s}$ is always rather less at NNLL than at NLL.

It is apparent from figure 4 that the difference between SCET and dQCD resummation, measured by $\boldsymbol{C}_{r}^{(2)}$, is sizable: at NNLO, it is of order $15 \%$ for the physical value of the Higgs mass, slowly decreasing as the Higgs mass increases towards the threshold - as one would expect, as in the threshold limit all different forms of resummation coincide by construction. This difference is as large as the effect of the resummation itself on the matched NNLO+NNLL result. Indeed, at the matched level, in dQCD resummation leads to an increase by about $10 \%$ of the unresummed NNLO result $[1,2]$. Using instead the SCET prediction with the scale choice eq. (3.1), and everything else being equal, we find that at the matched level the effect of resummation on the fixed-order result is negligible, at the level of a few percent. This fact is sometimes obscured, because in comparisons of SCET and dQCD different ingredients are often included in either calculation, instead of comparing like with like. 
However, if we look at $\boldsymbol{C}_{r}^{(1)}$, i.e., we tune all power suppressed terms in the SCET result so that it only differs by logarithmically subleading terms from the dQCD result, the difference is down to a few percent at NNLL, with SCET just above or just below the dQCD result, according to which choice is made for $\mu_{s}$. If finally we look at $\boldsymbol{C}_{r}^{(0)}$, i.e. the difference between $\mathrm{dQCD}$ and its $\mu_{s}$-dependent form, the difference is at the sub-percent level with any choice of $\mu_{s}$.

We conclude that the difference between what we have been calling respectively SCET and dQCD is large; however, it is almost entirely due to power-suppressed terms, which are really not a feature of either $\mathrm{dQCD}$ or SCET, but rather, an instance of the resummation ambiguities which are present in any approach and which have been previously discussed in refs. $[3,29,30]$. Indeed, as we have seen in section 2 , the leading $\mathcal{O}((1-z))$ power difference between the SCET and dQCD result can be expressed as a $\sqrt{z}$ prefactor, according to eq. (2.39). This prefactor (or rather, its $N$-space transformed version) is thus mostly responsible for the deviation of $\boldsymbol{C}_{r}^{(2)}$ from one, i.e. for the difference between the SCET and dQCD results. As already mentioned in section 2, this factor can be related to the collinear improvement discussed in refs. $[3,30]$, and thus the difference is akin to that between versions of resummation which do or do not include this collinear improvement.

Clearly, the size of the deviation measured by $\boldsymbol{C}_{r}^{(1)}$ will crucially depend on the choice of scale $\mu_{s}$. In particular, if $\mu_{s}=m_{H} / \bar{N}$, then $F\left(L, \mu_{s}\right)=1$ in eq. (2.20). Now, the high accuracy of the saddle-point approximation means that the Mellin inversion integral eq. (3.2) is completely dominated by $N=N_{0}(\tau)$. It follows that

$$
\mu_{s}=\frac{m_{H}}{\bar{N}_{0}(\tau)}
$$

is the optimal choice of scale if one wishes to remove the Landau pole from the dQCD resummed expression by replacing it with eq. (2.22), while leaving the result unchanged as much as possible. Equivalently, this is the choice that minimizes $\left|\ln \frac{m_{H}^{2}}{\mu_{s}^{2} N^{2}}\right|$, and thus maximizes the inequality eq. (2.18) which justifies this treatment of the Landau pole.

Also, this provides us with a criterion to judge whether any other (hadronic) choice of $\mu_{s}$ will lead to large or small deviation from the starting expressions. In refs. [5, 8, 9, 19] the function $g(\tau)$ which enters the scale choice eq. (3.1) is chosen as an ad-hoc functional form, whose parameters are determined, as already mentioned, by demanding that the one-loop contribution of $\tilde{s}_{\text {Higgs }}$ to the cross-section be minimized.

It is thus interesting to compare the choice of scale eq. (3.1), with $g(\tau)$ as in refs. [5, 8 , $9,19]$, with the "optimal" choice eq. (4.1). We do this in figure 5 , where, for reference, the naive hadronic scale choice which corresponds of taking $g(\tau)=1$ in eq. (3.1) is also shown. As already mentioned, in refs. $[5,8,9,19]$ two different ways to actually determine $g(\tau)$ are suggested: either by choosing the absolute minimum of the one-loop correction (referred to as $\mu_{s}^{I I}$ in figure 5), or starting from a high scale, and choosing $\mu_{s}$ as the value $\left(\mu_{s}^{I}\right)$ at which the one-loop term drops below $15 \%$. In either case, an empirical parametrization which approximately implements the condition is chosen. In figure 5 we show both $\mu_{s}^{I}, \mu_{s}^{I I}$, and also their average. 


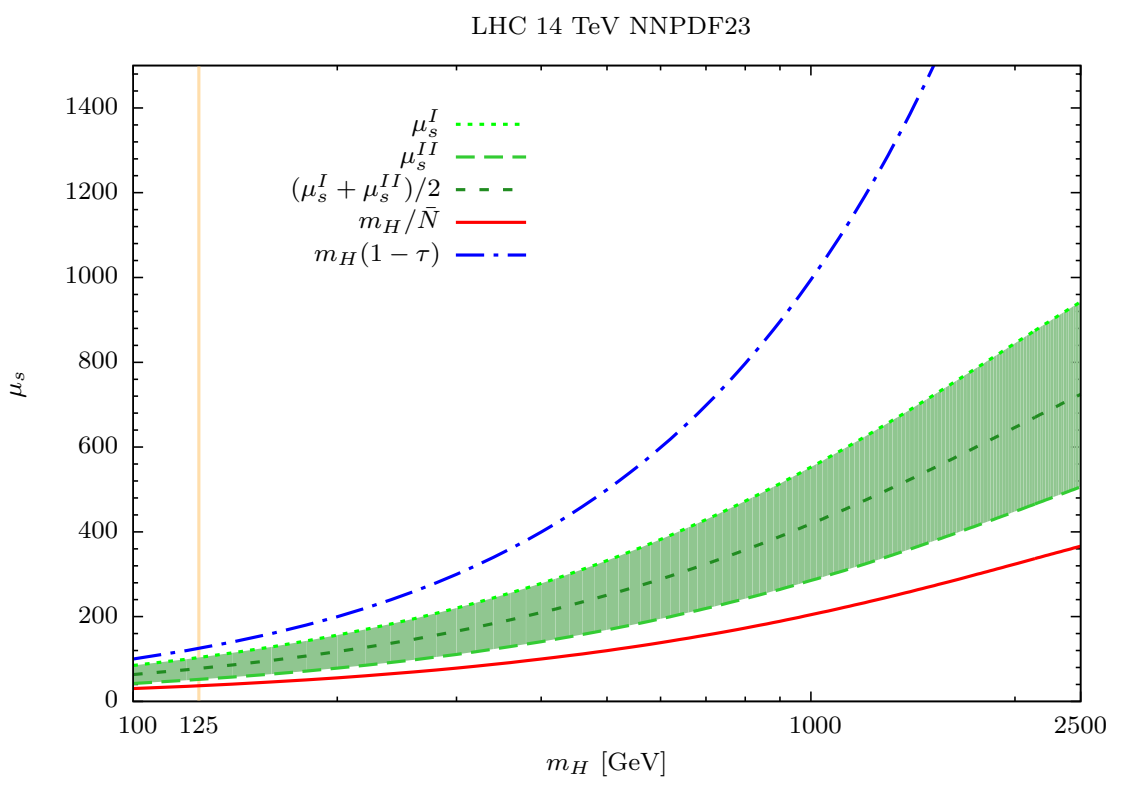

Figure 5. Comparison of choices of of soft scale $\mu_{s}$ : from bottom to top, the "optimal" scale choice eq. (4.1); eq. (3.1) with $g(\tau)$ determined as in refs. [6, 9, 19]; and the naive hadronic scale choice $\mu_{s}=m_{H}(1-\tau)$.

It is clear that the scale choice eq. (3.1) and the "optimal" choice eq. (4.1) are quite close, and rather different from the naive choice $\mu_{s}=m_{H}(1-\tau)$, which, of course, almost coincides with $m_{H}$ even for $m_{H}$ as large as $1 \mathrm{TeV}$. This explains the smallness of $\boldsymbol{C}_{r}^{(1)}$ seen in figure 4 , and also (since $m_{H} / \mu_{s} \sim 2$ ) why the resummation does have a visible effect, albeit not a huge one. The reason why these two scale choices are close is obvious: as we have seen, the optimal scale choice leads to the vanishing of all higher order contributions to $F\left(L, \mu_{s}\right)$. But $F\left(L, \mu_{s}\right)$ and $\tilde{s}_{\text {Higgs }}$ are closely related, see eq. (2.23): hence the condition of refs. $[5,8,9,19]$ is essentially an empirical version of the more quantitative condition eq. (4.1).

Before concluding, we would like to note that the actual implementation of resummation in dQCD ref. [2] and SCET refs. [6, 9, 19] differ in many further aspects. First of all, somewhat confusingly, a different nomenclature is used in the literature for the logarithmic accuracies of table 1: in particular, in refs. $[6,9,19]$ only the starred NLL*, NNLL*, etc., orders of resummation are considered, but they are referred to as NLL, NNLL etc. Specifically, the highest-order resummation considered in that reference is NNNLL*, which requires guessing the currently unknown $A_{4}$ coefficient (in refs. $[6,9,19]$ this is done by means of a Padé approximation). However, as shown in figure 1, NNNLL* resummation is almost indistinguishable from NNLL, so the extra effort of upgrading from NNLL to NNNLL* seems unwarranted, as results actually obtained using the RGHiggs code [32] at NNNLL (which is really NNNLL*) are very close to the NNLL, discussed here and shown in all plots of this paper.

Furthermore, the SCET resummation in refs. [6, 9, 19] also includes the exponentiation of a class of constant contributions proportional to $\pi^{2}$; the dQCD resummation also includes 
finite top and bottom mass effects [2], and so on. Some of these contributions (for instance, the $\pi^{2}$ exponentiation) have an effect which is quantitatively comparable to the overall effect of the resummation, and thus their inclusion can significantly affect results. They were all quantitatively assessed in the respective references, so there is no reason for us to discuss them here. Rather, by making sure that everything else is treated in the same way, we have focused on the effect due to the different treatment of logarithmically subleading and power-suppressed contributions.

\section{$5 \quad$ Summary and outlook}

We have provided a careful, step-by-step comparison of SCET and dQCD resummation, concentrating on differences in treatment of logarithmically subleading and power suppressed contributions. We have chosen Higgs production as a case study, but our results also hold for related processes such as Drell-Yan production. Also, we have considered a wide (unphysical) range of the Higgs mass, which extends to final states with a mass of several TeV.

Our main results can be summarized as follows (not necessarily in the order in which we have obtained them):

- The Landau pole can be removed from the dQCD resummed expression by introducing a dependence on an extra scale $\mu_{s}$. This scale may be chosen in an optimal way, such that the dependence on it is minimized, by using a saddle-point argument.

- The form of dQCD resummation thus obtained only differs from the SCET resummed result by logarithmically subleading and by power suppressed terms.

- A saddle-point argument allows for a comparison at the level of partonic cross-sections of different resummation schemes, even if the soft scale which is being resummed is chosen as a hadronic scale.

- Using this saddle-point argument, it can be shown that the fact that in the SCET result of refs. $[6,8,9,19]$ the soft scale $\mu_{s}$ depends on a hadronic scale (and thus the hadronic cross-section does not factorize upon Mellin transformation) has a negligibly small impact.

- The logarithmically subleading differences between the SCET and dQCD result are small if the scale is chosen according to the procedure suggested in refs. [6, 9, 19], which appears to be an empirically motivated version of the optimal dQCD scale choice referred to above.

- The power-suppressed differences between the SCET and dQCD result are large: in fact large enough that while NNLL resummation in dQCD leads to an increase of order of $\sim 10 \%$ of the NNLO cross-section when matched to it, in SCET it leads to an almost negligible increase, at the percent level. 
The fact that the large difference between SCET and dQCD resummation is dominated by power-suppressed terms means that, as mentioned in the introduction, the difference is really unrelated to the use of dQCD vs. SCET, and rather to known $[3,29,30]$ ambiguities when resummation is used to improve fixed-order computations away from threshold.

This might suggest the pessimistic conclusion that resummation, and more generally the soft approximation, do not provide reliable results in this kinematic region. This is of course a possible point of view, which, in particular, was recently advocated in ref. [31]. However we do not think that this is necessarily the case: rather, reliable results may be obtained, provided the choice of power-suppressed contribution is optimized by imposing requirements such as Mellin-space analiticity [3], which can be validated by comparison to the orders (up to NNLO) for which the full result is known. It is interesting to observe that if this is done, it turns out [38] that the effect of resummation on Higgs production in gluon fusion is in fact a further enhancement, which roughly doubles the enhancement found in the "standard" dQCD approach of ref. [2]. However, if this is not done the conclusion that the spread of resummed results is bigger than the impact of the resummation seems inevitable not only for Higgs production but also for processes which are much closer to threshold, such as, say production of a $Z^{\prime}$ with the mass of a few $\mathrm{TeV}$ at the LHC.

\section{Acknowledgments}

We thank G. Ferrera for asking a question which is answered by the argument presented in section 2, and S. Carrazza for help with NNPDF parton distributions. We thank the anonymous referee for very useful suggestions for the improvement of the paper. SF and GR are supported in part by an Italian PRIN2010 grant, and SF also by a European Investment Bank EIBURS grant, and by the European Commission through the HiggsTools Initial Training Network PITN-GA-2012-316704.

Open Access. This article is distributed under the terms of the Creative Commons Attribution License (CC-BY 4.0), which permits any use, distribution and reproduction in any medium, provided the original author(s) and source are credited.

\section{References}

[1] LHC Higgs Cross Section Working Group collaboration, S. Dittmaier et al., Handbook of LHC Higgs cross sections: 1. Inclusive observables, arXiv:1101.0593 [INSPIRE].

[2] D. de Florian and M. Grazzini, Higgs production at the LHC: updated cross sections at $\sqrt{s}=8$ TeV, Phys. Lett. B 718 (2012) 117 [arXiv:1206.4133] [INSPIRE].

[3] R.D. Ball, M. Bonvini, S. Forte, S. Marzani and G. Ridolfi, Higgs production in gluon fusion beyond NNLO, Nucl. Phys. B 874 (2013) 746 [arXiv:1303.3590] [InSPIRE].

[4] M. Bonvini, R.D. Ball, S. Forte, S. Marzani and G. Ridolfi, Updated Higgs cross section at approximate $N^{3} L O, J$. Phys. G 41 (2014) 095002 [arXiv: 1404.3204] [INSPIRE].

[5] V. Ahrens, T. Becher, M. Neubert and L.L. Yang, Origin of the large perturbative corrections to Higgs production at hadron colliders, Phys. Rev. D 79 (2009) 033013 [arXiv:0808.3008] [INSPIRE]. 
[6] V. Ahrens, T. Becher, M. Neubert and L.L. Yang, Renormalization-group improved prediction for Higgs production at hadron colliders, Eur. Phys. J. C 62 (2009) 333 [arXiv:0809.4283] [INSPIRE].

[7] V. Ahrens, T. Becher, M. Neubert and L.L. Yang, Updated predictions for Higgs production at the Tevatron and the LHC, Phys. Lett. B 698 (2011) 271 [arXiv:1008.3162] [INSPIRE].

[8] T. Becher, M. Neubert and B.D. Pecjak, Factorization and momentum-space resummation in deep-inelastic scattering, JHEP 01 (2007) 076 [hep-ph/0607228] [INSPIRE].

[9] T. Becher, M. Neubert and G. Xu, Dynamical threshold enhancement and resummation in Drell-Yan production, JHEP 07 (2008) 030 [arXiv:0710.0680] [INSPIRE].

[10] M. Bonvini, S. Forte, M. Ghezzi and G. Ridolfi, Threshold resummation in SCET vs. perturbative QCD: an analytic comparison, Nucl. Phys. B 861 (2012) 337 [arXiv: 1201.6364] [INSPIRE].

[11] M. Bonvini, S. Forte, M. Ghezzi and G. Ridolfi, The scale of soft resummation in SCET vs. perturbative QCD, Nucl. Phys. Proc. Suppl. 241-242 (2013) 121 [arXiv:1301.4502] [INSPIRE].

[12] G. Sterman and M. Zeng, Quantifying comparisons of threshold resummations, JHEP 05 (2014) 132 [arXiv: 1312.5397] [INSPIRE].

[13] L.G. Almeida et al., Comparing and counting logs in direct and effective methods of QCD resummation, JHEP 04 (2014) 174 [arXiv:1401.4460] [INSPIRE].

[14] S. Catani and L. Trentadue, Resummation of the QCD perturbative series for hard processes, Nucl. Phys. B 327 (1989) 323 [inSPIRE].

[15] G.F. Sterman, Summation of large corrections to short distance hadronic cross-sections, Nucl. Phys. B 281 (1987) 310 [InSPIRE].

[16] H. Contopanagos, E. Laenen and G.F. Sterman, Sudakov factorization and resummation, Nucl. Phys. B 484 (1997) 303 [hep-ph/9604313] [INSPIRE].

[17] S. Forte and G. Ridolfi, Renormalization group approach to soft gluon resummation, Nucl. Phys. B 650 (2003) 229 [hep-ph/0209154] [INSPIRE].

[18] S. Catani, M.L. Mangano, P. Nason and L. Trentadue, The resummation of soft gluons in hadronic collisions, Nucl. Phys. B 478 (1996) 273 [hep-ph/9604351] [INSPIRE].

[19] T. Becher and M. Neubert, Threshold resummation in momentum space from effective field theory, Phys. Rev. Lett. 97 (2006) 082001 [hep-ph/0605050] [INSPIRE].

[20] C.W. Bauer, S. Fleming and M.E. Luke, Summing Sudakov logarithms in $\vec{B} X_{S} \gamma$ in effective field theory, Phys. Rev. D 63 (2000) 014006 [hep-ph/0005275] [INSPIRE].

[21] C.W. Bauer, S. Fleming, D. Pirjol and I.W. Stewart, An effective field theory for collinear and soft gluons: heavy to light decays, Phys. Rev. D 63 (2001) 114020 [hep-ph/0011336] [INSPIRE].

[22] C.W. Bauer and I.W. Stewart, Invariant operators in collinear effective theory, Phys. Lett. B 516 (2001) 134 [hep-ph/0107001] [INSPIRE].

[23] C.W. Bauer, D. Pirjol and I.W. Stewart, Soft collinear factorization in effective field theory, Phys. Rev. D 65 (2002) 054022 [hep-ph/0109045] [INSPIRE]. 
[24] C.W. Bauer, S. Fleming, D. Pirjol, I.Z. Rothstein and I.W. Stewart, Hard scattering factorization from effective field theory, Phys. Rev. D 66 (2002) 014017 [hep-ph/0202088] [INSPIRE].

[25] A.V. Manohar, Deep inelastic scattering as $\vec{x} 1$ using soft-collinear effective theory, Phys. Rev. D 68 (2003) 114019 [hep-ph/0309176] [InSPIRE].

[26] B.D. Pecjak, Non-factorizable contributions to deep inelastic scattering at large $x$, JHEP 10 (2005) 040 [hep-ph/0506269] [INSPIRE].

[27] J. Chay and C. Kim, Deep inelastic scattering near the endpoint in soft-collinear effective theory, Phys. Rev. D 75 (2007) 016003 [hep-ph/0511066] [INSPIRE].

[28] A. Idilbi and X.-d. Ji, Threshold resummation for Drell-Yan process in soft-collinear effective theory, Phys. Rev. D 72 (2005) 054016 [hep-ph/0501006] [INSPIRE].

[29] M. Bonvini, S. Forte and G. Ridolfi, Soft gluon resummation of Drell-Yan rapidity distributions: theory and phenomenology, Nucl. Phys. B 847 (2011) 93 [arXiv:1009.5691] [INSPIRE].

[30] M. Krämer, E. Laenen and M. Spira, Soft gluon radiation in Higgs boson production at the LHC, Nucl. Phys. B 511 (1998) 523 [hep-ph/9611272] [INSPIRE].

[31] C. Anastasiou et al., Higgs boson gluon-fusion production at threshold in $N^{3} L O Q C D$, Phys. Lett. B 737 (2014) 325 [arXiv:1403.4616] [INSPIRE].

[32] http://rghiggs.hepforge.org/.

[33] C.F. Berger, C. Marcantonini, I.W. Stewart, F.J. Tackmann and W.J. Waalewijn, Higgs production with a central jet veto at NNLL+NNLO, JHEP 04 (2011) 092 [arXiv: 1012.4480] [INSPIRE].

[34] S. Forte, G. Ridolfi, J. Rojo and M. Ubiali, Borel resummation of soft gluon radiation and higher twists, Phys. Lett. B 635 (2006) 313 [hep-ph/0601048] [INSPIRE].

[35] M. Beneke, P. Falgari, S. Klein and C. Schwinn, Hadronic top-quark pair production with NNLL threshold resummation, Nucl. Phys. B 855 (2012) 695 [arXiv:1109.1536] [INSPIRE].

[36] M. Bonvini, S. Forte and G. Ridolfi, The threshold region for Higgs production in gluon fusion, Phys. Rev. Lett. 109 (2012) 102002 [arXiv:1204.5473] [INSPIRE].

[37] http://www.ge.infn.it/ bonvini/higgs/.

[38] M. Bonvini and S. Marzani, Resummed Higgs cross section at $N^{3} L L$, JHEP 09 (2014) 007 [arXiv: 1405.3654] [INSPIRE].

[39] R.D. Ball et al., Parton distributions with LHC data, Nucl. Phys. B 867 (2013) 244 [arXiv: 1207.1303] [INSPIRE]. 